\title{
Correction: Zainalabdeen, N., et al., Synthesis and Characterization of Naphthalenediimide-Functionalized Flavin Derivatives. Int. J. Mol. Sci. 2013, 14, 7468-7479.
}

Nada Zainalabdeen ${ }^{1}$, Brian Fitzpatrick ${ }^{1}$, Mohanad Mousa Kareem ${ }^{1}$, Vikas Nandwana ${ }^{2}$, Graeme Cooke $^{1, *}$ and Vincent M. Rotello ${ }^{2, *}$

1 Glasgow Centre for Physical Organic Chemistry, WestCHEM, School of Chemistry, University of Glasgow, Glasgow G12 8QQ, UK; E-Mails: n_y92@yahoo.com (N.Z.); brianf@chem.gla.ac.uk (B.F.); mohanad_1972@yahoo.com (M.M.K.)

2 Department of Chemistry, University of Massachusetts, Amherst, MA 01003, USA; E-Mail: nandwana@chem.umass.edu

* Authors to whom correspondence should be addressed;

E-Mails: Graeme.Cooke@glasgow.ac.uk (G.C.); rotello@chem.umass.edu (V.M.R.);

Tel./Fax: +44-141-330-5500 (G.C.).

Received: 2 July 2013; in revised form: 25 February 2014 / Accepted: 25 February 2014 / Published: 10 March 2014

In the original version of the manuscript [1] some of the analytical data for compounds $\mathbf{1}$ and $\mathbf{2}$ were incorrect. The correct NMR data are presented below. The authors apologize for any inconvenience this may have caused to the readers of this journal.

Compound 1:

${ }^{1} \mathrm{H}$ NMR (500 MHz, DMSO-d6) $\delta 11.64(\mathrm{~s}, 1 \mathrm{H}), 8.73$ (s, 4H), 8.57 (d, $\left.J=1.4 \mathrm{~Hz}, 1 \mathrm{H}\right), 8.16$ (dd, $J=8.9,1.4 \mathrm{~Hz}, 1 \mathrm{H}), 7.81(\mathrm{~d}, J=8.5 \mathrm{~Hz}, 2 \mathrm{H}), 7.64(\mathrm{~d}, J=8.5 \mathrm{~Hz}, 2 \mathrm{H}), 6.99(\mathrm{~d}, J=8.9 \mathrm{~Hz}, 1 \mathrm{H})$, $4.08(\mathrm{t}, J=7.0 \mathrm{~Hz}, 2 \mathrm{H}), 3.28(\mathrm{~m}, 2 \mathrm{H}), 1.69$ (quin, $J=7.0 \mathrm{~Hz}, 2 \mathrm{H}), 1.33(\mathrm{~m}, 8 \mathrm{H}), 0.86(\mathrm{t}, J=6.8 \mathrm{~Hz}$, $3 \mathrm{H}) .{ }^{13} \mathrm{C}$ NMR $(125 \mathrm{MHz}, \mathrm{DMSO}-\mathrm{d} 6) \delta 162.6(2 \mathrm{xC}=0), 162.3(2 \mathrm{xC}=0), 158.9,155.1,151.9,140.8$, 136.6, 136.1, 135.2, 133.7, 131.1 (2xC), 130.5 (4xC), 130.3 (q, $J=4 \mathrm{~Hz}), 128.6$ (q, $J=4 \mathrm{~Hz}), 128.4(2 \times C)$, 126.6, $126.5(2 \times C), 126.4(\mathrm{q}, J=31 \mathrm{~Hz}), 126.3(2 \mathrm{xC}), 126.2,123.2(\mathrm{q}, J=271 \mathrm{~Hz}), 117.8,39.9,30.9$, $28.5,28.3,27.1,26.3,21.9,13.7$.

Compound 2:

${ }^{1} \mathrm{H}$ NMR $\left(500 \mathrm{MHz}, \mathrm{CDCl}_{3}\right) \delta 8.77(\mathrm{~s}, 4 \mathrm{H}), 8.58(\mathrm{~d}, J=1.4 \mathrm{~Hz}, 1 \mathrm{H}), 8.03(\mathrm{dd}, J=9.1,1.4 \mathrm{~Hz}, 1 \mathrm{H})$, $7.87(\mathrm{~d}, J=8.4 \mathrm{~Hz}, 2 \mathrm{H}), 7.76(\mathrm{~d}, J=9.1 \mathrm{~Hz}, 1 \mathrm{H}), 7.27$ (d, $J=8.4 \mathrm{~Hz}, 2 \mathrm{H}), 5.37$ (s, 2H), 4.61 (br s, 
2H), 4.19 (t, 2H), 2.47 (sept, $J=6.7 \mathrm{~Hz}, 1 \mathrm{H}), 1.74(\mathrm{~m}, 2 \mathrm{H}), 1.47-1.23(\mathrm{~m}, 10 \mathrm{H}), 1.07$ (d, $J=6.7 \mathrm{~Hz}$, $6 \mathrm{H}), 0.87(\mathrm{t}, J=6.9 \mathrm{~Hz}, 3 \mathrm{H}) .{ }^{13} \mathrm{C} \mathrm{NMR}\left(125 \mathrm{MHz}, \mathrm{CDCl}_{3}\right) \delta 163.1(2 \mathrm{xC}=\mathrm{O}), 162.9(2 \mathrm{xC}=\mathrm{O})$, 159.0, 155.0, 149.9, 138.9, 137.5, 135.2, 134.9, 134.3, 131.7 (2xC), 131.5 (2xC), 131.2 (q, J=4 Hz), $131.1(4 \mathrm{xC}), 130.9$ (q, $J=4 \mathrm{~Hz}), 128.6(2 \mathrm{xC}), 127.1(2 \mathrm{xC}), 127.0$ (q, $J=28 \mathrm{~Hz}), 126.8(2 \times C)$, $123.1(\mathrm{q}, J=270 \mathrm{~Hz}), 116.9,51.5,44.9,41.2,31.9,29.4,29.3,28.2,27.6,27.2,22.8,20.2(2 \times C), 14.2$.

The corrected version of the paper can be accessed at http:/www.mdpi.com/1422-0067/15/3/4255/s1.

\section{Reference}

1. Zainalabdeen, N.; Fitzpatrick, B.; Kareem, M.M.; Nandwana, V.; Cooke, G.; Rotello, V.M. Synthesis and characterization of naphthalenediimide-functionalized flavin derivatives. Int. J. Mol. Sci. 2013, 14, 7468-7479.

(C) 2014 by the authors; licensee MDPI, Basel, Switzerland. This article is an open access article distributed under the terms and conditions of the Creative Commons Attribution license (http://creativecommons.org/licenses/by/3.0/). 